Acta Crystallographica Section D

Biological Crystallography

ISSN 0907-4449

\title{
Crystallization and preliminary crystallographic studies of antibacterial polypeptide LCI expressed in Escherichia coli
}

LCI is a type of novel antibacterial polypeptide secreted by a Bacillus subtilis strain. It consists of 47 residues with a molecular weight of $5468 \mathrm{Da}$. Using bioengineering, LCI was expressed in Escherichia coli DH5 $\alpha$ with recombinant plasmid pBVAB16. It was crystallized using PEG 4000 as a precipitant. The crystal belongs to space group $P 6_{2} 22$ or $P 6_{4} 22$, with unit-cell parameters $a=b=29.30, c=187.09 \AA$, and diffracts to $2.44 \AA$. A set of diffraction data to $2.8 \AA$ was collected.
Received 28 June 2001 Accepted 15 October 2001 \section{Huan-Chen Wang, Qing Xu, Zhang-Liang Chen and Guang-Ying Lu*}

Wei-Bin Gong, Yu-He Liang,

National Laboratory of Protein Engineering and Plant Genetic Engineering, College of Life Sciences, Peking University, Beijing 100871, People's Republic of China

Correspondence e-mail: lugy@pku.edu.cn

C 2001 International Union of Crystallography Printed in Denmark - all rights reserved

\section{Introduction}

Antibacterial proteins are a class of very important proteins that exist widely in the bodies or secretions of plants (Roberts \& Selitrennikoff, 1986), microorganisms (Von Tersch \& Carlton, 1983), insects (Okada \& Natori, 1985) and animals (Scheit et al., 1985). Liu et al. (1990) screened and isolated a B. subtilis strain (named A014) whose secretions possess very strong antibacterial activities, especially against the pathogen (Xanthomonas campestris pv. oryzea) of rice leaf-blight disease, which is a serious threat to rice production and causes great losses in yields in most rice fields annually. Until now, there has been no efficient method to control this disease. It was found that the antibacterial functions are carried out by several types of protein in the secretions (Liu et al., 1991, 1992, 1993). The proteins are named LCI (Liu et al., 1990), LCII (Liu et al., 1992) and LCIII (Liu et al., 1993), respectively, according to the order of their elution peaks from a CM-52 chromatographic column. The sequence of LCI was determined by protein sequencing. LCI consists of 47 residues, with a molecular weight of 5468 and a pI of 10.25 . No homologous protein was found in the protein information resources the Protein Sequence Database (PIR-PSD) and the Protein Data Bank (PDB).

After incubation for $20 \mathrm{~min}$ at 333, 353 or $373 \mathrm{~K}$, LCI retains $100,85.3$ or $12.5 \%$ of its antibacterial activity, respectively (Liu et al., 1990), indicating that LCI is thermostable and that its three-dimensional structure may be highly stable, although there is no cysteine in its sequence and hence no disulfide bonds in the structure. Owing to the low yield of LCI secreted by wild B. subtilis A014, it is difficult to carry out crystallographic studies. Recently, LCI was expressed in large quantities with an E. coli expression system using DH5 $\alpha$ with the recombinant plasmid pBVAB16 in our laboratory. Its molecular weight is $5464 \mathrm{Da}$ as measured by mass spectrometry and it shows comparable thermal stability and antibacterial activities to the native protein (to be published elsewhere). The present work reports the crystallization and preliminary crystallographic studies of the antibacterial polypeptide LCI expressed by engineering E. coli. with DH5 $\alpha$. Study of its three-dimensional structure will be helpful in revealing the mechanisms of its antibacterial activities and the thermal stability for the protein.

\section{Methods and results}

Crystallization was carried out using the hanging-drop vapour-diffusion method at $293 \mathrm{~K}$. A $11 \mu \mathrm{l}$ droplet containing $3.64 \mathrm{mg} \mathrm{ml}^{-1}$ LCI, $3.09 \%(w / v) \quad$ PEG 4000, $0.55 \%(v / v)$ dioxane and $0.01 M$ NaOAc-HOAc buffer $\mathrm{pH}$ 5.7 was equilibrated against $0.4 \mathrm{ml}$ reservoir solution containing 34\%(w/v) PEG 4000 in double-distilled water. Crystals were obtained with maximum dimensions of $0.3 \times 0.1 \times$ $0.1 \mathrm{~mm}$ after $7-10 \mathrm{~d}$ (Fig. 1). Only twin crystals were obtained in the absence of dioxane in the droplet. A crystal was mounted in a glass capillary and X-ray diffraction data were collected on a MAR 345 image-plate system using $\mathrm{Cu} K \alpha(\lambda=1.5418 \AA)$ radiation from a sealed-tube generator operated at $50 \mathrm{kV}$ and $40 \mathrm{~mA}$ with a crystal-to-detector distance of $200 \mathrm{~mm}$. The data were collected at $293 \mathrm{~K}$. The scan range was $90^{\circ}$ with an oscillation of $1^{\circ}$. The data were processed using the programs DENZO and SCALEPACK (Otwinowski \& Minor, 1997). The crystal diffracts to $2.44 \AA$ and the effective resolution is $2.8 \AA$. A total of 27210 observations of 1506 unique reflections within the resolution range 30.0-2.80 $\AA$ were collected with an $R_{\text {merge }}$ of $10.9 \%$ (35.9\% in the 


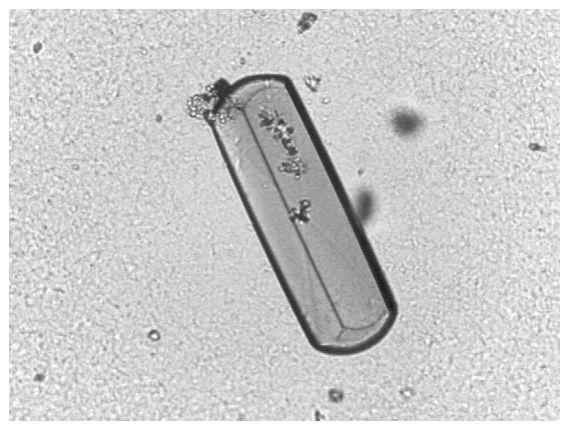

Figure 1

The crystal of antibacterial polypeptide LC1 expressed in E. coli. The longest dimension is approximately $0.3 \mathrm{~mm}$.

highest resolution shell 2.87-2.80 $\mathrm{A}$ ) and a multiplicity of 7.65 (7.47 in the highest resolution shell $2.87-2.80 \AA$ ). The completeness of the overall data set is $99.8 \%$ $(100.0 \%$ in the highest resolution shell 2.87-2.80 А). The percentage of the overall data set with $I>3 \sigma(I)$ is $85.2 \%$ and with
$I>2 \sigma(I)$ is $89.1 \%$. The crystal belongs to one of the enantiomorphous space groups $P 6_{2} 22$ or $P 6_{4} 22$. The unit-cell parameters are $a=b=29.30, c=187.09 \AA$, with a $\chi^{2}$ of 0.751. Assuming one LCI molecule per asymmetric unit cell, the calculated $V_{\mathrm{M}}$ is $2.12 \AA^{3} \mathrm{Da}^{-1}$, corresponding to a solvent content of $39.7 \%$ (Matthews, 1968). The values lie within the normal range for protein crystals. As there is no homologous protein available whose three-dimensional structure has been determined, the preparation of heavy-atom derivatives and further crystallographic studies are under way.

We would like to thank Dr Xu-Dong Zhao and Dr Cai-Hong Yun for help in data collection at Institute of Biophysics, Academia Sinica. This work was supported by a research grant provided by National Natural Science Foundation of China (No. 39670160), a research grant provided by the
National Laboratory of Biomacromolecules, Institute of Biophysics, Academia Sinica and a project grant provided by the National Manned Space Engineering of China.

\section{References}

Liu, J. Y., Li, Z., Pan, N. S. \& Chen, Z. L. (1992). Chin. J. Biotechnol. 8, 266-270.

Liu, J. Y., Liu, W., Pan, N. S. \& Chen, Z. L. (1991). Acta Bot. Sin. 33, 157-161.

Liu, J. Y., Pan, N. S. \& Chen, Z. L. (1990). Rice Genet. Newsl. 7, 151-154.

Liu, J. Y., Pan, N. S. \& Chen, Z. L. (1993). Studies on Plant Genetic Engineering, edited by Z.-L. Chen, pp. 300-305. Beijing: Peking University Press.

Matthews, B. W. (1968). J. Mol. Biol. 33, 491-497. Okada, M. \& Natori, S. (1985). J. Biol. Chem. 260, 7174-7177.

Otwinowski, Z. \& Minor, W. (1997). Methods Enzymol. 276, 307-326.

Roberts, W. K. \& Selitrennikoff, C. P. (1986). Biochim. Biophys. Acta, 880, 161-170.

Scheit, K. H., Shivaji, S. \& Bhargava, P. M. (1985). J. Biochem. (Tokyo), 97, 463-471.

Von Tersch, M. A. \& Carlton, B. C. (1983). J. Bacteriol. 155, 872-877. 